\title{
Aplicación de la termografía sobre dron a la evaluación de la eficiencia del riego por aspersión.
}

\author{
Cristina Jarén Morilla ${ }^{1}$, Salvatierra Bellido Benito ${ }^{1}$, Manuel López Rodríguez $^{1}$
}

Instituto de Investigación y Formación Agraria de Andalucía, Rancho de la Merced (IFAPA, sede Chipiona-Cádiz; cristina.jaren@juntadeandalucia.es, benito.salvatierra@juntadeandalucia.es, manuel.lopez.rodriguez@juntadeandalucia.es

Resumen: En las instalaciones de riego, el control de la uniformidad en el agua aplicada da una medida (más o menos directa) sobre los caudales en cada parte de la superficie cultivada, la falta de uniformidad en el riego causa elevadas pérdidas de agua por percolación y la generación de contaminación difusa. En la mayoría de las ocasiones el objetivo elegido es determinar si los caudales y presiones son uniformes (no varían o cuanto varían) entre un sector y otro de la instalación de riego, sin considerar en su totalidad otros elementos, como el suelo y el clima, que pueden afectar esta uniformidad. Tradicionalmente el uso de los pluviómetros así como la realización de medidas de humedad directamente en el suelo transformados en coeficientes que permiten cuantificar esta uniformidad. Estos métodos, salvo que se trate de pequeñas instalaciones, se aplican sobre una muestra de la misma siendo sus resultados extensibles al total. El uso de cámaras termográficas, ahora cada vez más asequible economicamente puede ser una herramienta útil para medir de forma rápida y precisa en toda la superficie regada.

Partiendo de parcelas con bajo grado de humedad en el suelo se ha comparado las medidas teóricas y de pluviómetros y muestreo en parcelas regadas por aspersores, con los resultados obtenido mediante la técnnica termografica sobre dron, en diferentes circunstancias de tiempo de riego.

Los resultados obtenidos nos muestran que el método es viable y de gran aplicabilidad para evaluar la eficiencia del uso del agua en las parcelas regadas, mejorando la misma en el diseño y manejo delriego y disminuyendo con ello los impactos negativos en el medio.

Palabras clave: Riego 1; coeficiente de uniformidad 2; termografía 3; medio-ambiente 4. 


\title{
Application of thermography on drone to the evaluation of the effi- ciency of sprinkler irrigation.
}

\section{Cristina Jarén Morilla ${ }^{1}$, Salvatierra Bellido Benito $^{1}$, Manuel López Rodríguez ${ }^{1}$}

1 Instituto de Investigación y Formación Agraria de Andalucía, Rancho de la Merced (IFAPA, sede Chipiona-Cádiz; cristina.jaren@juntadeandalucia.es, benito.salvatierra@juntadeandalucia.es, manuel.lopez.rodriguez@juntadeandalucia.es

\begin{abstract}
In irrigation facilities, the control of the uniformity in the applied water gives a measure (more or less direct) on the flows in each part of the cultivated area, the lack of uniformity in the irrigation causes high losses of water by percolation and the generation of diffuse pollution. In most cases, the objective chosen is to determine if the flows and pressures are uniform (they do not vary or how much they vary) between one sector and another of the irrigation installation, without fully considering other elements, such as soil and climate., which can affect this uniformity. Traditionally the use of rain gauges as well as the realization of humidity measurements directly in the soil transformed into coefficients that allow to quantify this uniformity. These methods, except in the case of small installations, are applied to a sample of the same, their results being extensible to the total. The use of thermal imaging cameras, now more and more economically affordable, can be a useful tool to measure quickly and accurately on the entire irrigated area.
\end{abstract}

Starting from plots with a low degree of humidity in the soil, theoretical measurements and rain gauges and sampling in plots watered by sprinklers have been compared with the results obtained through the thermographic technique on drone, in different circumstances of irrigation time.

The results obtained show us that the method is viable and highly applicable to evaluate the efficiency of the use of water in irrigated plots, improving it in the design and management of irrigation and thereby reducing the negative impacts on the environment

Keywords: Irrigation 1; uniformity coefficient 2; thermography 3; environment 4. 


\section{Introducción}

En las instalaciones de riego, la uniformidad da una medida (más o menos directa) sobre los caudales aplicados en cada parte del cultivo. En la mayoría de las ocasiones el objetivo elegido es determinar si los caudales y presiones son uniformes (no varían o cuanto varían respecto a la medida) entre un sector y otro de la instalación de riego, como medida de la uniformidad del agua aplicada.

Tradicionalmente el uso de los pluviómetros y su tratamiento han permitido obtener unos coeficientes que cuantifican esta uniformidad. Los parámetros más utilizados son el coeficiente de variación(CV), la uniformidad de distribución referida al 25\% del área menos regada (UD) y el coeficiente de Uniformidad (CU) de Christiansen (1942). Este último es el que con más frecuencia se utiliza en el riego por aspersión (Dechmi,F. Et al), aunque en nuestro ámbito la uniformidad de distribución aparece con más frecuencia.

La interpretación de los mismos varía según las fuentes, pero una aproximación normalmente aceptada sería para la UD aplicado a los sistemas de riego localizados se admite que valores de $90 \%-100 \%$ la uniformidad es excelente; $80 \%$ - 90\% uniformidad buena; 70\% - 80\% aceptable; $<70 \%$ inaceptable. En el caso de los riegos por aspersión, un coeficiente de uniformidad $>80 \%$ indicaría una uniformidad de distribución buena. Para este tipo de sistemas de riego, Keller y Bliesner (1990) consideran hasta un CU del $75 \%$ como relativamente baja. Según Cuenca (1989), la eficienca potencial de las coberturas totales estaría entre un $70 \%$ en diseños para bajo viento y un $82 \%$ en diseños para alto viento.

Andalucía es la Comunidad Autónoma con mayor superficie ${ }^{1}$ de regadío 29,18\% (1.117.858 has) del total nacional. De esta superficie sólo el 11,67\% (65.992 has) se riega por aspersión. Pero su uso se concentra en zonas donde predominan los cultivos herbáceos anuales llegando a superar el $50 \%$ de la superficie regada (ejem. Marismas de Lebrija).

En el riego por aspersión la toma de muestra para evaluar la uniformidad es complicada y costosa sobre todo en grandes instalaciones agrícolas. Estos métodos en el mejor de los casos y salvo que se trate de pequeñas instalaciones, hacen su aplicación sobre una muestra de la misma en el tiempo y el espacio, siendo sus resultados extensibles al total del tiempo de duración del cultivo y a la superficie ocupada, presentando complicaciones en la representatividad y la interpretación de la muestra.

La termografía es una técnica que permite determinar temperaturas a distancia y sin necesidad de contacto físico con el objeto a estudiar. Desarrollada en el S.XX, es ya habitual en los sectores industriales, de construcción y energéticos. Recientemente, la incorporación de cámaras termográficas a los drones está poniendo estas técnicas al alcance de un mayor número de sectores, aprovechando la mejora de tecnología y el abaratamiento de costes de la misma, abriendo un campo de aplicaciones muy interesante en la agricultura. 
En este ensayo se aplican las técnicas de termografía sobre dron para la evaluación de la uniformidad de un riego por aspersión en condiciones de campo.

\section{Materiales y métodos.}

Los ensayos se realizaron sobre dos parcela al aire libre con suelo artificial enarenado, en la finca del Instituto de Investigación y Formación Agraria y Pesquera (IFAPA) de la Junta de Andalucía en su centro de Chipiona. Se regó con agua superficial suministrada por la CRR Costa Noroeste de Cádiz, en varias pruebas entre los meses de mayo a septiembre del año 2021 .

La instalación de riego por aspersión con un marco de 12×12 metros, (siendo ésta la unidad de comparación) constaba de aspersores Unirain F46 con boquilla 9/64 con vaina prolongadora, situados a 0,8 m sobre el suelo, una presión de trabajo 250 y $450 \mathrm{kPa}$ y tiempos de riego de $1 \frac{1}{2}$ y 1 hora

Se dispuso de una cámara térmica Zenmuse H20T (La carcasa del cardán DJI Zenmuse H20T se compone de cuatro sensores separados: Cámara gran angular: un sensor CMOS de 1/2,3 pulgadas que emite $12 \mathrm{MP}$ con un campo de visión de 82,9 $9^{\circ}$. Cámara con zoom: un sensor CMOS de $20 \mathrm{MP}$ de 1/1,7 pulgadas que incluye un zoom óptico híbrido x23 (200x máximo) que ofrece $4 \mathrm{~K} 30 \mathrm{p}$ en video. Telémetro láser: permite medir la distancia de un objeto desde el dron de 3 a 1200 metros con una precisión de unos 0,2 metros. Sensor térmico radiométrico: la resolución de 640 por 512 píxeles ofrece una frecuencia de actualización de 30p y tiene una sensibilidad térmica de $\leq 50 \mathrm{mk@f1.0} \mathrm{(NEDT).).} \mathrm{La} \mathrm{misma} \mathrm{va} \mathrm{montada} \mathrm{sobre} \mathrm{un} \mathrm{dron,} \mathrm{Martice}$ 300 RTK (para aplicaciones industriales. Ofrece hasta 55 minutos de vuelo, un intervalo de transmisión máximo de hasta $8 \mathrm{~km}$, funciones avanzadas de IA, 6 sensores direccionales y de posicionamiento.)

Se calculó la curva característica del aspersor tipo elegido en una instalación aislada del propio centro y tomando la caracterización del mismo en trabajos de más profuniddad realizados en el propio centro (Salvatierra, B et alt, 2010)

Sobre dos parcelas de 12×12 metros secas en profundidad se dio un riego, desde los vértices de la misma, de media y una hora de duración. Se realizaron controles con pluviómetros de la lluvia caída en la parcela, 31 pluviómetros uniformemente distribuidos, con un diámetro de abertura de 0,15 m y 0,17 m de altura. Con barrenas se controló la penetración del agua en el suelo de las parcelas muestreando 100 puntos en cada una de ellas, en una cuadrícula de 1,5x1,5 m. Y por último se tomaron imágenes con dron de las parcelas, antes y después del riego, con un pixel de 0,038 x 0,038 m. de resolución, aproximadamente 99.000 datos muestreados de la parcela de $12 \times 12$.

\section{Resultados y discusión}




\section{Congreso Nacional de Riegos CARTAGENA 2021}

En una instalación aislada del viento se obtuvo la curva característica del aspersor utilizado, en dos regímenes de presión, 250 y 450 $\mathrm{kPa}$, Figura nำ.

Con la curva característica se calculó la superficie mojada teórica y la lluvia recibida en la misma, definiendo perímetros mojados de 1,52,5-4-6-9-12-14 metros de radio y sus intersecciones, y presiones de trabajo de 250 y $450 \mathrm{kPa}$., Figuras 2 y 3 .

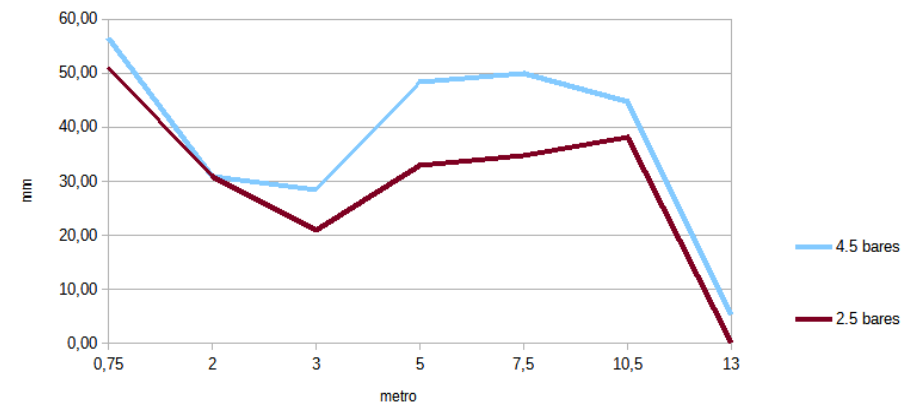

Figura 1. Aspersor UNIRAIN F46 con boquilla 9/64 y vaina prolongadora
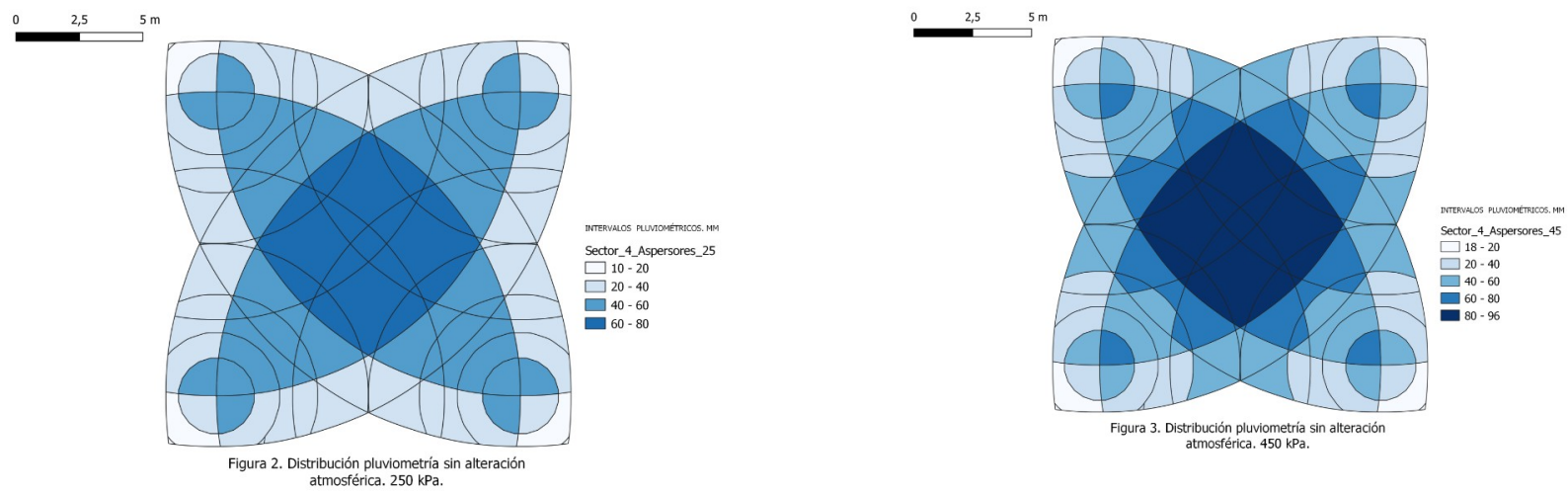

El coeficiente de uniformidad de Christiansen teórico fue de 74,15 y el 73,98\% y una uniformidad de distribución de 63,71 y 67,71 \% para las presiones de 250 y $450 \mathrm{kPa}$ respectivamente. Logicamente valores bajos pero que se justifican por lo reducido de la parcela (4 aspersores) y el acusado efecto borde.

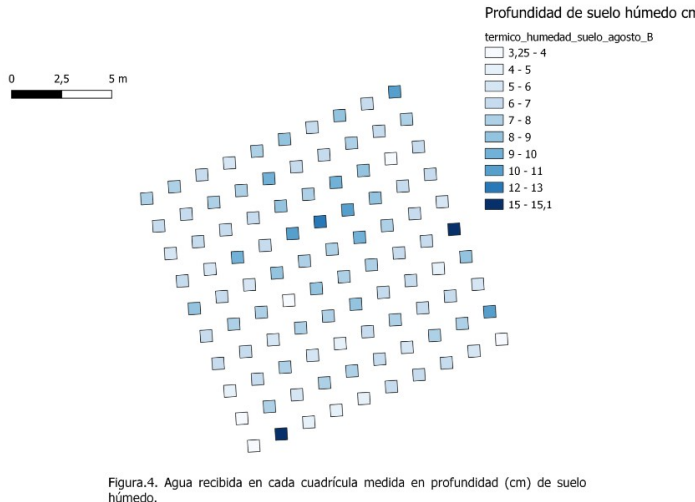

Se tomaron muestras del suelo húmedo después del riego en las dos parcelas ( $1 / 2$ y 1 hora), Figura. 4 para $1 / 2$ hora, midiendo el peso del perfil húmedo y la profundidad alcanzada por el frente húmedo, después del riego.

Los coeficientes obtenidos fueron para $1 / 2$ y 1 hora respectivamente CU $79,88 \%$ y UD 70,19\%, mientras que en la segunda parcela los valores fueron de $71,34 \%$ y $62,79 \%$. 
Se obtuvo el coefiente de uniformidad a partir de pluviómetros distribuidos en las dos parcelas regadas. Respectivamente los valores para cada coeficiente fueron CU 61,47 y $74,52 \%$ y UD 54,36 y $69,26 \%$. Valores más bajos de los normales para los aspersores y boquillas utilizados, pero como se dijo, justificados teniendo en cuenta las dimensiones del ensayo y el efecto borde acumulado respecto a una instalación de tamaño mayor.

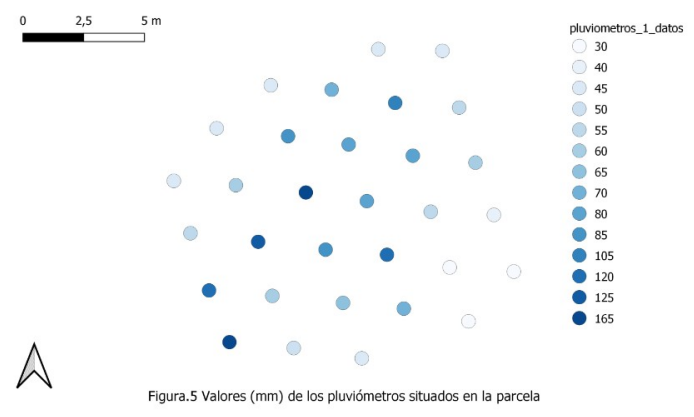

Para las imágenes de termografía tomadas (Figuras 6, 7 y 8), la distribucion de las temperaturas tomadas en cada pixel $(0,035 \times 0,035 \mathrm{~m})$ de la imagen comprendida entre los aspersores, se muestra en la figura 9.
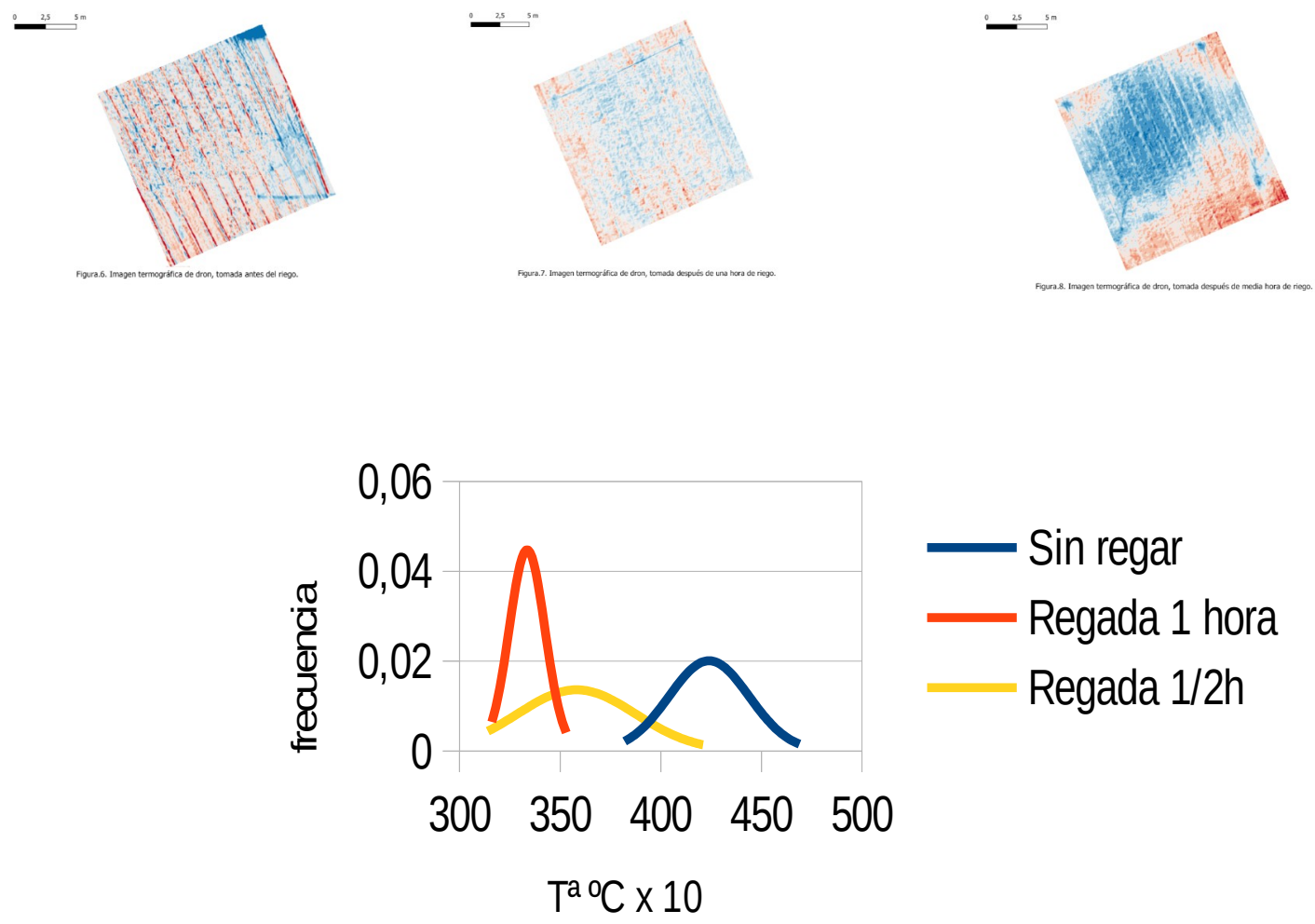

Figura.9. Distribución de valores de la termografía. 
Tabla 1. Valores termográficos de las imágenes según tratamiento

\begin{tabular}{cccc}
\hline Tratamiento & P. sin riego & $\begin{array}{c}\text { P. 1 hora de } \\
\text { riego }\end{array}$ & $\begin{array}{c}\text { P. 1/2 hora de } \\
\text { riego }\end{array}$ \\
\hline $\mathrm{T}^{\mathrm{a}}$ promedio & 42,3 & 33,3 & 35,8 \\
Desv. estandart & 19,8 & 8,9 & 29,3 \\
\hline
\end{tabular}

Tabla 2. Valores de coeficientes obtenidos según tratamiento

\begin{tabular}{ccccc}
\hline Método & Teóricas & $\begin{array}{c}\text { Pluvió } \\
\text { metros }\end{array}$ & $\begin{array}{c}\text { Humedad } \\
\text { de suelo }\end{array}$ & $\begin{array}{c}\text { Termografía } \\
\mathbf{1} \text { 2_1 horas }\end{array}$ \\
\hline CU de Christiansen & $74 \%$ & $61-74 \%$ & $71-79 \%$ & $92 \_98 \%$ \\
UD q q $25 \%$ & $63-68 \%$ & $54-69 \%$ & $63-71 \%$ & $88 \_96 \%$ \\
\hline
\end{tabular}

El balance de energía en la superficie de un suelo está fuertemente influenciada por la posición diurna del Sol, por la composición del suelo, la labor y por el contenido de agua entre otros.

El balance de energía, Radiación neta del suelo $\left(\mathrm{R}_{\mathrm{ns}}\right)$ se expresa como:

$$
R_{n s}=H_{s}+G_{s}+\lambda E_{s}
$$

donde $H_{s}$ es el calor sensible, $G_{s}$ calor del suelo y calor latente $\lambda E_{s}$ en un suelo seco este término sería nulo.

Si se riega en un momento determinado del día en un suelo desnudo homogéneo (composición, color, humedad, labores,...) y condiciones microclimáticas iguales (no sombras, cortavientos, etc...), la zona regada experimentará una variación de la temperatura respecto a la no regada debido a una variación del calor sensible (al estar el agua a diferente temperatura que el suelo) y a las pérdidas de calor latente. Estos cambios energéticos pueden ser medidos en términos de variación de temperatura de la superficie del suelo.

Una vez que el riego ha concluido, si el mismo ha sido eficaz, ha garantizado que en toda la superficie se haya depositado una cantidad suficiente de agua (figura 7), esto hace que la superficie del suelo pase de una $T_{\text {as }}$ a una menor temperatura $T_{\text {ah }}$ (figura 9). Si el riego no es homogéneo y no deposita la misma cantidad de agua en un punto del suelo que en otro, antes de su saturación superficial (menor tiempo de riego) se provoca un gradiente de temperaturas más disperso (figura 8), siendo esta medida un buen indicador de 


\section{Congreso Nacional de Riegos CARTAGENA 2021}

la mayor o menor bondad del sistema de riego, pudiendo resalizarse esta medida en la totalidad de la superficie de riego y no sólo en una muestra, sin alterar la superficie de cultivo.

\section{Conclusiones}

Este sistema es una herramienta asequible y fácil de implementar para evaluar la eficiencia de un sistema de riego por aspersión, proporcionando un conocimiento que permita minimizar la lisiviación y las zonas subregadas, actuando sobre las pérdidas de nutrientes y una minimización de la llegada de éstos al ciclo del agua. Al poder análisar el total de la superficie en riego, puede ayudar a evaluar tanto el diseño como el manejo de la instalación de riego por aspersión. La rapidez y comodidad de su uso permite repetir las medidas en diferentes condiciones climáticas (viento) mejorando la eficiencia de estos sistemas de riego por aspersión.

La dispersión que se obtiene en los primeros estadíos del riego debe ser una buena herramienta para comparar diferentes instalaciones, manejo y diseño, del riego por aspersión.

\section{Referencias}

1. Christiansen J.E., (1942). Irrigation by Sprinkling. Univ. Of California. Agric. Exp. Sta. Bull. 670, 124pp.

2. Dechmi, F. (P), Playán Jubillar, E., Faci González, J.M., Bercero, A. Uniformidad de los Sistemas de riego por aspersión en el polígono de la Loma de Quinto (Zarragoza). Unidad de Suelos y riegos, Laboratorio de Agronomía y Medio Ambiente. SIG-DGA Zaragoza.

3. Cuenca, R.H. (1989). Irrigation system design: an ingeneering approaxh. Englewood Cliffs, New Jersey, Prentice-Hall, Inc. 552 pp.

4. Keller, J \& Bliesner, RD. (1990). Sprinkle and Trickle Irrigation. AVI Book. Van Nostrand Reinhold. New York. USA.

5. 1. Análisis de los Regadíos Españoles. Año 2020. Fuente: Encuesta sobre Superficies y Rendiemintos de Cultivos (ESCYRCE).

6. Salvatierra, B. 2010. Uniformidad de riego de las instalaciones de riego por aspersión, Boletín trimestral de Información al Regante. № 14 . Enero, marzo, pp 7-8.

7. Salvatierra, B. 2012. Evaluaciones y manejo de las instalaciones de riego por aspersión en la remolacha azucarera en Andalucía. Revista AIMCRA 111, pp 3-9.

8. Salvatierra-Bellido, B., Montero-Martínez, J., Pérez-Urrestarazu, L. 2018. Development of an automatic test bench to assess sprinkler irrigation uniformity in different wind conditions. Computers and Electronics in Agriculture 151, 31-40. doi: 10.1016/j.compag.2018.05.036

9. Rafael Fernández Gómez, Nicolás A. Oyonarte Gutiérrez, Juan P. García Bernal,, Mª del Carmen Yruela Morillo, Mercedes Milla Milla, Ricardo Ávila Alabarces, Pedro Gavilán Zafra, 2010. Manual de Riego para Agricultores Módulo 3: Riego por Aspersión. Junta de Andalucia.

10. Laserna Arcas, S.1, Montero Martínez, J. 2, Sheikhesmaeili, O. 3, Alcázar Bascuñana, E.4. análisis de la uniformidad de riego en Sistemas de aspersión semiportátil con aspersores de gran tamaño. 